Rev. Saúde públ., S. Paulo, 19:270-7, 1985.

\title{
SITUAÇÃO ATUAL DA ESQUISTOSSOMOSE MANSONI NO LAGO DA PAMPULHA, BELO HORIZONTE, MG, BRASIL*
}

\author{
Omar dos Santos Carvalho** \\ Carlos Tito Guimarães** \\ Cristiano Lara Massara** \\ João Eduardo Ribeiro Bonésio***
}

\begin{abstract}
CARVALHO, O. dos $\mathbf{S}$. et al. Situação atual da esquistossomose mansoni no Lago da Pampulha, Belo Horizonte, MG. Brasil. Rev. Saúde públ., S. Paulo, 19:270-7, 1985. 1985.

RESUMO: Descreve-se a atual distribuição dos planorbídeos hospedeiros intermediários do Schistosoma mansoni no Lago da Pampulha, Belo Horizonte, MG (Brasil) e a rápida dispersão de Biomphalaria tenagophila por todo o perímetro lacustre. Relatase o primeiro encontro de $B$. tenagophila naturalmente infectada por $S$. mansoni, naquela coleção hídrica. Dišute-se, a importância epidemiológica destas observações e a possibilidade da $B$. tenagophila vir a substituir a primitiva população de $B$. glabrata no Lago da Pampulha.
\end{abstract}

UNITERMOS: Schistosoma mansoni. Biomphalaria glabrata. Biomphalaria tenagophila. Lago da Pampulha, Belo Horizonte, MG, Brasil.

\section{INTRODUÇÃO}

A história da esquistossomose mansoni em Belo Horizonte (Minas Gerais, Brasil) está intimamente relacionada com a bacia hidrográfica do Lago da Pampulha, ainda que os primeiros casos autóctones desta parasitose tivessem sido diagnosticados em $1919^{26}$, ou seja, 17 anos antes do início da construção da barragem, que daria origem ao lago, em 1936. A referida coleção hídrica foi, no passado, responsabilizada pela provável infecção de centenas de pessoas que dele se utilizaram (Martins e Versiani ${ }^{15}$, 1939). Segundo esses mesmos autores, foi após a criação daquele lago que o número de portadores de Schistosoma mansoni começou a aumentar "extraordinariamente" na capital mineira.

O Lago da Pampulha, distando cerca de $9 \mathrm{~km}$ do centro comercial de Belo Horizonet, e utilizado para práticas de natação, remo, esqui e pescarias, é re- sultante do represamento de sete córregos (Mergulhão, Tijuco, Ressaca, Água Funda, Baraúna, AABB e Olhos d'água). De formato amebóide, com cerca de $21 \mathrm{~km}$ de perímetro, ocupa uma área de $2,8 \mathrm{~km}^{2}$, abrigando um volume d'água estimado em 13 milhões de $\mathrm{m}^{3}$.

$\mathrm{O}$ primeiro relato sobre a ocorrência de planorbídeos hospedeiros intermediários de $S$. mansoni no interior do lago foi feito por Martins e Versiani ${ }^{14}$, em 1938. Naquela oportunidade foram coletados "numa extensão de cerca de 400 metros", 42 exemplares de Biomphalaria glabrata, sendo que um $(2,4 \%)$ eliminava cercárias de $S$. mansoni.

Colocada a par do problema, a Prefeitura de Belo Horizonte, entre outras medidas, determinou o esvaziamento temporário daquela coleção hídrica. Naquela oportunidade, relatam Martins e Versiani ${ }^{15}$ (1939), "fizemos um cálculo gros-

- Trabalino parcialmente financiado pelo Conselho Nacional de Desenvolvimento Científico e Tecnológico (CNFq) e Frefeitura Municipal de Belo Forizonte.

" Do Centro de Pesquisas "René Rachou" (CPqRR) - FIoCRUz - Av. Augusto de Lima, 1715 30000 - Belo Horizonte, MG - Brasil

** Estagíário do CPqRR - FIOCRUZ. 
CARVALHO, $O$. dos $S$. et al. Situação atual da esquistossomose mansoni no Lago da Pampulha, Belo Horizonte, MG, Brasil. Rev. Saúde públ., S. Paulo, 19:270-7, 1985.

seiro da quantidade de "Planorbis" ali existente, tomando por base o seu número médio por metro quadrado e a área abrangida pela represa: verificamos que chegavam a 12 ou 15 milhóes aproximadamente".

Em 1940-41, Martins (cf. Milward-deAndrade, $1969^{19}$ ), realizando uma "inspeção sumária" no lago verificcu que o número de planorbídeos era bastante reduzido. Cerca de 11 anos mais tarde, Martins e Falcão ${ }^{13}$ (1953) capturaram, naquela coleção hídrica, 176 exemplares de $B$. glabrata, dos quais somente um $(0,6 \%)$ eliminava cercárias de $S$. mansoni.

Em maio de 1954, a barragem primitiva rompeu-se, sendo reconstruída e reinaugurada em janeiro de 1958. A partir de 1979, a Prefeitura de Belo Horizonte iniciou a construção de uma ilha, na enseada do córrego Ressaca, para instalação de uma grande área de lazer, promovendo alterações sensíveis no lago. Em outubro de 1982, a Superintendência de Desenvolvimento da Pesca - SUDEPE (Regional de Minas Gerais) deu início a uma série de peixamentos no lago, com espécies alóctones, visando, entre outros objetivos, promover o controle biológico dos hospedeiros intermediários da esquistossomose mansoni.

A fauna malacológica do Lago da Pampulha, relatada até o momento, é composta por: planorbídeos (B. glabrata, $B$. tenagophila, $B$. schrammi, Depanotrema cimex, D. Lucidum); ampularídeos (Pomacea haustrum); ancilídeos (Ancylus sp); fisídeos (Physa sp); limineídeos (Lymnaea $s p$ ) e esferídeos (Pisidium sp) (Martins e Versiani ${ }^{14}$, 1938; Milwardde-Andrade, 1920, 1969, 1972; Carvalho, $1974^{2}$; Milward-de-Andrade e Carvalho ${ }^{21}, 1978$ ).

No presente estudo são apresentados dados recentes sobre a distribuição espacial dos planorbídeos hospedeiros intermediários de $S$. mansoni no interior do lago e o relato, pela primeira vez, de $B$. tenagophila naturalmente infectada por $S$. mansoni naquela coleção hídĩica.

\section{MATERIAL E METODOS}

No período de maio de 1981 a abril de 1984 , foram realizadas dez coletas de planorbídeos ao longo das margens do Lago da Pampulha. Em seis oportunidades (agosto e setembro/81; janeiro e maio/82; janeiro e novembro/83), as capturas foram realizadas em sete estações, abrangendo $500 \mathrm{~m}$ para cada lado das margens, a partir da desembocadura dos córregos formadores do lago. Realizaram-se ainda quatro pesquisas (maio/ 81; junho/82; abril/83 e abril/84), em toda a orla lacustre, que foi previamente dividida em 32 estações de pesquisa com cerca de $500 \mathrm{~m}$ cada uma.

Em 22 de fevereiro de 1984 realizouse, na enseada do Córrego Zoológico, uma captura para obtenção de planorbídeos para estudos experimentais em laboratório.

As coletas foram realizadas utilizando-se concha metálica com $25 \mathrm{~cm}$ de altura, com perfurações de cerca de $0,5 \mathrm{~cm}$ de diâmetro, nas laterais e no fundo, adaptada a uma haste de madeira com cerca de $1,20 \mathrm{~m}$ de comprimento.

Adotou-se o critério de mergulhar a concha na coleção hídrica, a cada passo. Os moluscos recolhidos eram acondicionados em sacos plásticos e, posteriormente, examinados em laboratório, por compressão entre lâminas de vidro, sob observação microscópica.

\section{RESULTADOS}

Os levantamentos malacológicos proporcionaram a coleta de 4.162 exemplares de planorbídeos. Deste total, 3.695 pertenciam à espécie $B$. tenagophila, sendo que entre estes um $(0,03 \%)$ eliminava cercárias de $S$. mansoni, e 467 eram B. glabrata, dos quais dois $(0,43 \%)$ eliminavam cercárias do trematódeo em questão (Tabela 1). 
CARVAlHO, O. dos $S$. et al. Situação atual da esquistossomose mansoni no Lago da Pampulha, Belo Horizonte, MG, Brasil. Rev. Saúde públ., S. Paulo, 19:270-7, 1985.

TABELA 1

Resultados das pesquisas de planorbídeos no Lago da Pampulha, Belo Horizonte, MG (Brasil) Maio/81 a abril $/ 84$

\begin{tabular}{lcccc}
\hline & \multicolumn{2}{c}{ Biomphalaria glabrata } & \multicolumn{2}{c}{ Biomphalaria tenagophila } \\
\cline { 2 - 5 } Data & \multicolumn{2}{c}{ Positivas para } & Positivas para \\
& Capturadas & S. mansoni & Capturadas & S. mansoni \\
\hline Maio/81* & 21 & $1(4.8)$ & 203 & 0 \\
Agosto & 0 & 0 & 69 & 0 \\
Setembro & 0 & 0 & 247 & 0 \\
Janeiro/82 & 3 & 0 & 76 & 0 \\
Maio & 0 & 0 & 86 & 0 \\
Junho* & 11 & 0 & 328 & 0 \\
Janeiro/83 & 187 & $1(0.5)$ & 414 & 0 \\
Abril* & 192 & 0 & 319 & 0 \\
Novembro & 39 & 0 & 840 & 0 \\
Abril/84* & 14 & 0 & 1.113 & $1(0.09) * *$ \\
\hline Total & 467 & $2(0.4)$ & 3.695 & $1(0.03)$ \\
\hline
\end{tabular}

( ) Percentagem de infecção.

* Pesquisa realizada em todo o perímetro lacustre.

* $O$ exemplar de $B$. tenagophila encontrado infectado em $22 / 2 / 84$ não consta da Tabela, pois o mesmo foi obtido de material coletado para estudos laboratoriais.

A B. tenagophila foi capturada em 31 das 32 estações distribuídas ao longo das margens do lago, enquanto $B$. glabrata foi coletada em 14 estações (Figura).
Por outro lado, na coleta realizada em 22 de fevereiro de 1984 , foram obtidos 188 exemplares de $B$. tenagophila, entre os quais um $(0,53 \%)$ encontrava-se pa-

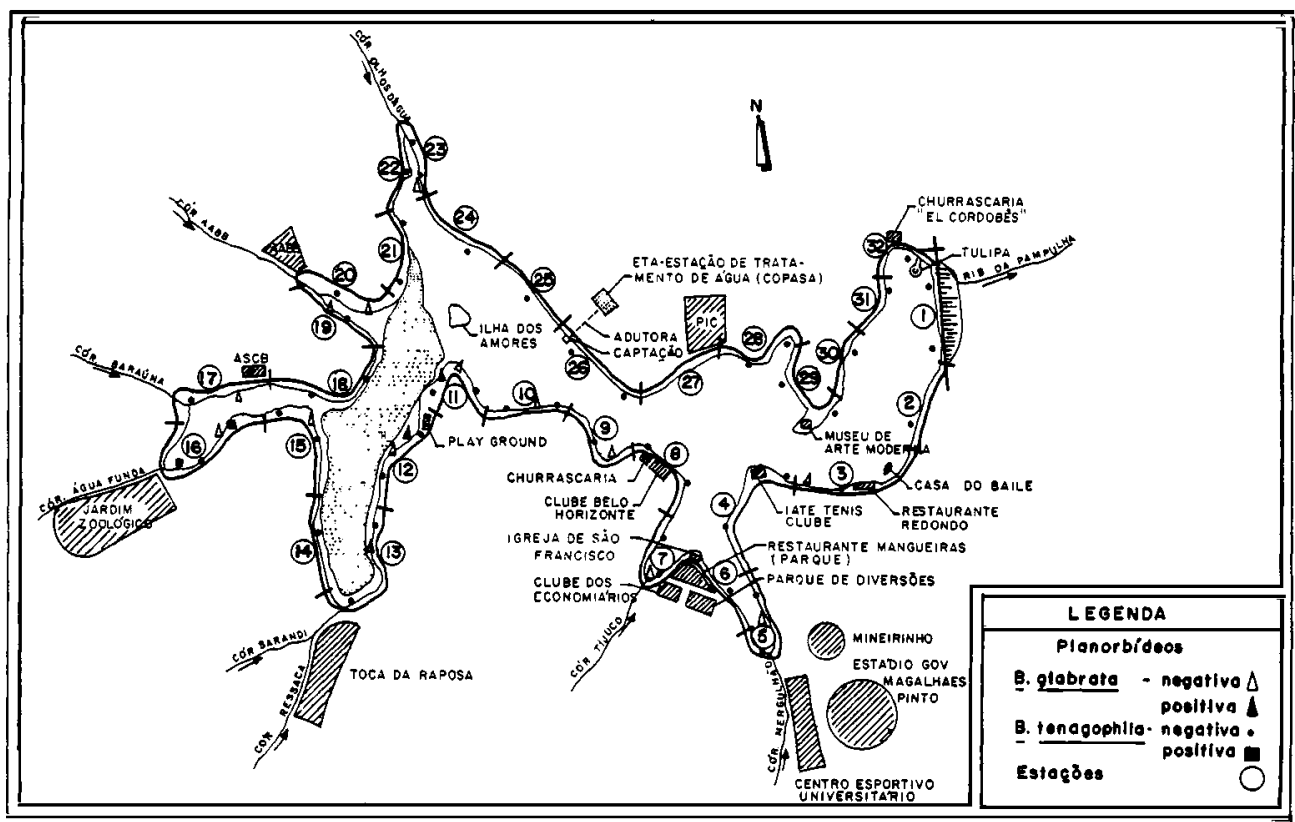

Figura: Lago da Pampulha. 
CARVALHO, O. dos $S$. et al. Situação atual da esquistossomose mansoni no Lago da Pampulha, Belo Horizonte, MG, Brasil. Rev. Saúde públ., S. Paulo, 19:270-7, 1985.

rasitado por $S$. mansoni, constituindo-se no primeiro relato de $B$. tenagophila, naturalmente infectada, em Belo Horizonte, ou mais precisamente no interior do Lago da Pampulha.

\section{DISCUSSÃO}

Revendo a literatura sobre a ocorrência de hospedeiros intermediários de
S. mansoni, no interior do Lago da Pampulha, verifica-se que após a inauguração da atual barragem em janeiro de 1958 até abril de 1981 , a população de planorbídeos dentro do lago manteve-se escassa, não sendo descrito nenhum exemplar parasitado por $S$. mansoni (Tabela 2).

\section{TABELA 2}

Levantamentos malacológicos realizados no Lago da Pampulha, Belo Horizonte, MG (Brasil), segundo diferentes autores (1938-1984)

\begin{tabular}{|c|c|c|c|c|c|}
\hline \multirow[b]{2}{*}{ Autor } & \multirow[b]{2}{*}{ Data } & \multicolumn{2}{|c|}{ Biomphalaria glabrata } & \multicolumn{2}{|c|}{ Biomphalaria tenagophila } \\
\hline & & Capturadas & $\begin{array}{l}\text { Positivas } \mathrm{p} / \\
\text { S. mansoni }\end{array}$ & Capturadas & $\begin{array}{l}\text { Positivas } \mathrm{p} / \\
\text { S. mansoni }\end{array}$ \\
\hline Martins e Versiani 14 & 1938 & 42 & $1(2,4 \%)$ & 0 & 0 \\
\hline Martins e cols. ${ }^{13}$ & 1953 & 176 & $1(0,6 \%)$ & 0 & 0 \\
\hline Milward-de-Andrade 18 & 1959 & 118 & 0 & 0 & 0 \\
\hline Milward-de-Andrade 19 & 1969 & 427 & 0 & 0 & 0 \\
\hline Milward-de-Andrade 20 & 1972 & 0 & 0 & 28 & 0 \\
\hline Carvalho 2 & 1974 & 64 & 0 & 35 & 0 \\
\hline Carvalho e cols. ${ }^{6}$ & 1975 & 64 & 0 & -35 & 0 \\
\hline $\begin{array}{l}\text { Milward-de-Andrade e } \\
\text { Carvalho21 }\end{array}$ & 1978 & 64 & 0 & 35 & 0 \\
\hline Guimarães 11 & $1978(1)$ & 442 & 0 & 1.380 & 0 \\
\hline Carvalho e Guimarães 3 & $1984(2)$ & 414 & $2(0,5 \%)$ & 1.742 & 0 \\
\hline
\end{tabular}

(1) Capturas realizadas no periodo de 1970 a 1976

(2) Capturas realizadas no período de 1981 a 1983.

Em 1972, Milward-de-Andrade ${ }^{20}$ relata, pela primeira vez, a existência de $B$. tenagophila no Lago da Pampulha (outubro/70). Naquela ocasião, foram coletados "numa área não maior que um metro quadrado da barragem propriamente dita daquele lago (cuja extensão é de cerca de 500 metros), 28 exemplares pigmentados de $B$. tenagophila". Esta população de $B$. tenagophila permaneceu, por algum tempo, confinada às imedia- ções da barragem, onde foi originalmente observada.

$O$ encontro de quatro exemplares de planorbídeos parasitados por $S$. mansoni (dois $B$. glabrata e dois $B$. tenagophila) e a dispersão atual desta última espécie, por todo o perímetro lacustre, traduzem a mais importante alteração ocorrida nos últimos 30 anos, da história natural da esquistossomose mansoni no Lago da Pampulha. A constatação destes fatos 
CARVAlHo, O. dos S. et al. Situação atual da esquistossomose mansoni no Lago da Pampulha, Belo Horizonte, MG, Brasil. Rev. Saúde públ., S. Paulo, 19:270-7, 1985.

deve merecer atenção especial das autoridades responsáveis, face a relatos recentes de exemplares de $B$. tenagophila infectados, em duas cidades do Estado de Minas Gerais: Jaboticatubas (Melo e col. $^{16,17}, 1982$, 1983) e Itajubá (Carvatho e col. ${ }^{8}, 1984$ ).

Por outro lado, estudos experimentais, envolvendo $B$. tenagophila procedentes de diversas localidades do Estado de Minas Gerais, têm demonstrado a suscetibilidade deste planorbídeo a diversas cepas de $S$. mansoni (Carvalho e col. ${ }^{7}$, 1979; Carvalho e Souza ${ }^{4,5}, 1979,1980$; Corrêa e col. ${ }^{9}$, 1979; Paraense e Corrêa ${ }^{23}$, 1978; Santos e col. ${ }^{24}, 1979$; Souza e col. ${ }^{25}, 1983$ ).

Em decorrência da ocupação de quase toda a orla do Lago da Pampulha pela $B$. tenagophila, encontram-se em curso observações mensais, com 0 intuito de verificar a ocorrência de um processo competitivo entre a espécie recém-introduzida e a primitiva população de $B$. glabrata.

A hipótese de exclusão competitiva entre espécies de planorbídeos foi sugerida por Paraense ${ }^{22}$ (1970), ao observar o deslocamento de uma população de $B$. glabrata, cinco anos após a introdução de $B$. tenagophila numa lagoa existente no Bairro Baleia, em Belo Horizonte, MG. Observações semelhantes foram registradas por Barbosa ${ }^{1}$ (1973), nos arredores de Recife (PE) e por Kawazoe ${ }^{12}$ (1980), no município de Ourinhos, SP.

O Lago da Pampulha tem sido objeto de profundas alteraçōes ecológicas, em virtude da interferência humana, sem os necessários estudos prévios, ou mesmo avaliação das possíveis conseqüências futuras.

Em junho de 1979, a Prefeitura Municipal de Belo Horizonte iniciou a construção de uma ilha, na enseada do Cór- rego da Ressaca, para a instalação de uma grande área de lazer. Para sua execução foi necessário um volume de terra da ordem de $2.200 .000 \mathrm{~m}^{3}$, removidos através de dragagem com draga flutuante*.

Essa movimentação de terra, além de favorecer o aparecimento de novos criadouros de planorbídeos nos canais que circundam a ilha, veio comprometer ainda mais o processo de assoreamento, por que passa o Lago da Pampulha, decorrente de esgotos, material arenoso e outros fragmentos rochosos transportados pelos sete córregos tributários, ou pelas chuvas que carreiam para seu interior material das áreas adjacentes (Milward-deAndrade ${ }^{19}, 1969$ ).

Decorridos cerca de seis anos do seu início, a obra se encontra no mais completo abandono, observando-se, ainda, intensa atividade clandestina de extração de areia no seu interior.

Em três oportunidades, a Sudepe introduziu no Lago da Pampulha cerca de 3.000 alevinos de espécies alóctones de peixes, ou seja: carpa (Cyprinus carpio), curimatã (Prochilodus scrofa), dourado (Salminus brasiliensis), mandi (Pimelodus maculatus), matrinchã (Brycon lundii e $B$. hilari), piau verdadeiro (Leporinus obtusidens), piau três pintas (L. reinhardt), tilápia (Sarotherodon niloticus) e trairão (Hoplias lacerdae). Os três peixamentos foram realizados: 2/out./82, 4/dez./82 e $15 /$ fev. $/ 84$ com exemplares oriundos da Estação de Hidrobiologia e Psicultura de Furnas (Centrais Elétricas de Minas Gerais), localizada em Conceição das Alagoas, MG, e do Parque Municipal de Belo Horizonte. Estão ainda previstos dois outros peixamentos com exemplares originários de Conceição das Alagoas e da Estação Central de Psicultura de Itiuba (CONDEVASP),

* Dados fornecidos pelo Departamento Nacional de Obras e Saneamento - DNOS (9. ${ }^{a} \mathrm{Di}$ retoria Regional) em 07/janeiro/1985. 
CARVALHO, O. dos S. et al. Situação atual da esquistossomose mansoni no Lago da Pampulha, Belo Horizonte, MG, Brasil. Rev. Saúde públ., S. Paulo, 19:270-7, 1985.

município de Porto Real do Colégio, Estado de Alagoas*.

Em decorrência dos peixamentos realizados, verificou-se uma afluência significativa de pescadores, sobretudo das áreas circunvizinhas, que são vistos diariamente em suas águas. Este fato poderá ter grande importância epidemiológica e enfatiza a necessidade de atenção para o problema da esquistossomose no Lago da Pampulha, face a presença de indivíduos parasitados por $S$. mansoni adaptados a $B$. tenagophila ali existentes.

Outro aspecto relevante nesses peixamentos, a merecer medidas adequadas de vigilância epidemiológica, é a possibilidade de introdução de espécies alóctones de planorbídeos, através do carreamento de desovas ou mesmo de exemplares jovens ou adultos, juntamente com peixes, quando estes não são submetidos à quarentena ou não são originários de estações sabidamente isentas de moluscos (Corrêa e cols. ${ }^{10}, 1970$ ).

\section{AGRADECIMENTOS}

Ao Dr. Wladimir Lobato Paraense, do Centro Internacional de Malacologia do Instituto Oswaldo Cruz - FIOCRUZ; e ao Sr. Carlos Rubens Teixeira da Silva, do Centro de Pesquisas "René Rachou" - FIOCRUZ, pela identificação específica dos moluscos. Aos Professores Amilcar Viana Martins e Dirceu Wagner Carvalho de Souza, pela revisão do texto e sugestões apresentadas. Aos Srs. José Geraldo Amorim da Silva e Antonio Carlos do Prado (CPqRR-FIOCRUZ) pela assistência técnica.

CARVALHO, O. dos $S$. et al. [The present position regarding schistosomiasis mansoni in Pampulha lake, Belo Horizonte, MG, Brazill. Rev. Saúde públ., S. Paulo, 19:270-7, 1985.

ABSTRACT: The present distribution of planorbid snails, the intermediate hosts of Schistosoma mansoni, in Pampulha lake (Belo Horizonte, Minas Gerais - Brazil) and the rapid dispersal of Biomphalaria tenagophila in the lake perimeter are describ. ed. The presence of $B$. tenagophila naturally infected by $S$. mansoni in this hydric collection is also reported for the first time. The epidemiological importance of these observations is indicated as also is the possibility of $B$. tenagophila replacing the primitive population of $B$. glabrata in the Pampulha lake.

UNITERMS: Schistosoma mansoni. Biomphalaria glabrata. Biomphalaria tenagophila. Pampulha lake, Belo Horizonte, MG, Brazil.

\section{REFERENCIAS BIBLIOGRÁFICAS}

1. BARBOSA, F.S. Possible competitive displacement and evidence of hybridization between two Brazilian species of planorbid snails. Malacologia, 14:401-8, 1973.

2. CARVALHO, O. dos S. Roedores silvestres na epidemiologia da esquistossomose mansoni no Lago da Pampulha, Belo Horizonte, Minas Gerais (Brasil) com especial referência ao Holochilus brasiliensis (Rodentia, Cricetidae). Belo Ho- rizonte, 1974. [Dissertação de Mestrado - Instituto de Ciências Biológicas da UFMG].

3. CARVALHO, O. dos S. \& GUIMARÃES, C.T. Distribuição atual dos planorbídeos hospedeiros intermediários de Schistosoma mansoni Sambon, 1907, no Lago da Pampulha, Belo Horizonte, MG (Brasil). In: Congresso da Sociedade Brasileira de Medicina Tropical, 20. ${ }^{\circ}$, Salvador, 1984. São Paulo, 1984. p. 81.

* Dados fornecidos pela Coordenadoria Regional da Superintendência de Desenvolvimento da Pesca - SUDEPE, em Minas Gerais, em 30/3/1984 
CARVALHO, O. dos S. et al. Situação atual da esquistossomose mansoni no Lago da Pampulha, Belo Horizonte, MG, Brasil. Rev. Saúde públ., S. Paulo, 19:270-7, 1985.

4. CARVALHO, O dos S. \& SOUZA, C.P. Comportamento de Biomphalaria tena. gophila (D'Orbigny, 1835) de Itajubá (MG, Brasil), exposta a cepa "SJ" de Schistosoma mansoni Sambon, 1907. In: Congresso da Sociedade Brasileira de Parasitologia, 4. ${ }^{\circ}$ Campinas, 1979. Campinas, 1979. p. 105.

5. CARVALHO, O. dos S. \& SOUZA, C.P. Suscetibilidade de Biomphalaria tenagophila (D'Orbigny, 1853), originárias de Ravena, Mun. de Sabará, MG (Brasil), a cepa "LE" de Schistosoma mansoni. In: Congresso da Sociedade Brasileira de Parasitologia, 5. ${ }^{\circ}$, Rio de Janeiro, 1980. Rio de Janeiro, 1980. p. 152.

6. CARVALHO, O. dos $S$. et al. Roedores silvestres na epidemiologia da esquistossomose mansônica no Lago da Pampulha, Belo Horizonte, Minas Gerais (Brasil). Rev. Soc. bras. Med., 9(1): 27-35, 1975.

7. CARVAlHo, O. dos S. et al. Suscetibilidade de Biomphalaria tenagophila (D’Orbigny, 1835), de Itajubá (MG), à infecção pela cepa "LE" de Schistosoma mansoni Sambon, 1907, de Belo Horizonte, MG (Brasil). Rev. Saúde públ., S. Paulo, 13:20-5, 1979.

8. CaRValho, O. dos $S$. et al. Primeiro encontro de Biomphalaria tenagophila (D'Orbigny, 1835) naturalmente infectada, com Schistosoma mansoni, em Itajubá, sul do Estado de Minas Gerais, Brasil. Rev. Saúde públ., S. Paulo, 19:88-91, 1985.

9. CORREA, M.C.R, et al. Suscetibilidade de linhagens de Biomphalaria tenagophila e Biomphalaria glabrata a duas cepas de Schistosoma mansoni (LE Belo Horizonte, MG e SJ - São José dos Campos, SP). Rev. Inst. Med. trop. S. Paulo, 21(2):72-6, 1979.

10. CORREA, R.R. et al. Dispersão de Biomphalaria straminea, hospedeira intermediária do Schistosoma mansoni, através da distribuição de peixes. Rev. Saúde públ., S. Paulo, 4:117-27, 1970.

11. GUIMARÃES, C.T. Observações bio-ecológicas sobre Pomacea haustrum (Reeve, 1856): sua utilização no controle bioló- gico da esquistossomose mansoni. Belo Horizonte, 1978. [Dissertação de Mestrado - Instituto de Ciências Biológicas da UFMG].

12. KAWAZOE, U. Competição biológica entre Biomphalaria glabrata (Say, 1818) e Biomphalaria tenagophila (D'Orbigny, 1835 ), em criadouros naturais no município de Ourinhos, SP (Brasil). Rev. Saúde públ., S. Paulo, 14: 65-87, 1980.

13. MARTINS, A.V. \& FALCÃO, A.L. Distribuição geográfica dos planorbídeos e seus índices de infestação pelas cercárias de Schistosoma mansoni no município de Belo Horizonte. [Apresentado ao $10 .^{\circ}$ Congresso Brasileiro de Higiene, Curitiba, 1953 - Mimeografado].

14. MARTINS, A.V. \& VERSIANI, W. Schistosomose mansoni em Belo Horizonte. Brasil méd., 52: 471-2, 1938.

15. MARTINS, A.V. \& VERSIANI, W. Plano de combate a "Schistosomose mansoni" em Belo Horizonte. Hospital, Rio de Janeiro, 15(3): 197-206, 1939

16. MELO, A.L. et al. Sobre o encontro de Biomphalaria tenagophila naturalmente infectada com Schistosoma mansoni no município de Jaboticatubas, Minas Gerais. In: Congresso da Sociedade Brasileira de Parasitologia, $7 .^{\circ}$, Porto Alegre, 1982. Porto Alegre, 1982. p. 63.

17. MELO, A.L. et al. Infecão experimental persistente de Biomphalaria tenagophila pelo Schistosoma mansoni em Jaboticatubas, Minas Gerais. In: Congresso da Sociedade Brasileira de Parasitologia, 8\%/Jornada Paulista de Parasitologia, 5. a, São Paulo, 1983. São Paulo, 1983. p. 53.

18. MILWARD-DE-ANDRADE, $R$. O problema da esquistossomose mansoni no lago artificial da Pampulha, Belo Horizonte, Minas Gerais (Brasil), Rev. bras. Malar., 10: 653-74, 1959.

19. MILWARD-DE-ANDRADE, R. Nota ecológica sobre o Lago da Pampulha (Belo Horizonte, MG), com especial referência aos planorbídeos (Pulmonata, planorbidae). Rev. bras. Malar., 21: 59-116, 1969. 
CARVALHO, O. dos S. et al. Situação atual da esquistossomose mansoni no Lago da Pampulha, Belo Horizonte, MG, Brasil. Rev. Saúde públ., S. Paulo, 19:270-7, 1985.

20. MILWARD-DE-ANDRADE, R. Primeiro encontro de Biomphalaria tenagophila (D'Orbigny, 1835) no Lago da Pampulha, Belo Horizonte, MG. Cienc. Cult., 24(supl.): $375,1972 . \quad\left[24 .^{2}\right.$ Reunião Anual da Sociedade Brasileira para o Progresso da Ciência, São Paulo, 1972 - Resumo].

21. MILWARD-DE-ANDRADE, R. \& CARVALHO, O. dos S. Fauna planorbídica da Bacia hidrográfica do Lago da Pampulha, MG (Brasil) (Pulmonata, planorbidae). In: Congresso da Sociedade Brasileira de Medicina Tropical, 14. $/$ Congresso da Sociedade Brasileira de $\mathrm{Pa}$ rasitologia, 3.․, João Pessoa, 1978. Resumo dos temas livres. João Pessoa 1978. p. 89.

22. PARAENSE, W.L. Planorbídeos hospedeiros intermediários do Schistosoma mansoni. In: Cunha, A.S., ed. Esquistossomose mansoni. São Paulo, Ed. USP, 1970. p. 13-30.
23. PARENSE, W.L. \& CORREA, L.R. Differential susceptibility of Biomphalaria tenagophila populations to infection with a strain of Schistosoma mansoni. J. Parasit., 64: 822-6, 1978.

24. SANTOS, M.B.L. et al. Suscetibilidade ao Schistosoma mansoni de híbridos de Biomphalaria tenagophila do Taim, RS, Cabo Frio, RJ e Belo Horizonte, MG. Rev. Inst. Med. trop. S. Paulo, 21: 281-6, 1979

25. SOUZA, C.P. et al. Suscetibilidade de Biomphalaria tenagophila de Belo Horizonte e adjacências à infecção com três cepas de Schistosoma mansoni. Rev. Inst. Med. trop. S. Paulo, 25: 168-72, 1983.

26. TEIXEIRA, J.M. A schistosomose mansônica na infância em Belo Horizonte. Belo Horizonte, Imprensa Oficial, 1921.

Recebido para publicação em 11/06/1984

Reapresentado em 16/04/1985

Aprovado para publicação em 19/04/1985 